Honam Mathematical J. 36 (2014), No. 1, pp. 113-129

http://dx.doi.org/10.5831/HMJ.2014.36.1.113

\title{
A NEW APPROACH FOR CHARACTERIZATION OF CURVE COUPLES IN EUCLIDEAN 3-SPACE
}

\author{
Siddika Özkaldi Karakuş, Kazim İlarslan*, and Yusuf Yayli
}

\begin{abstract}
In this study, we have investigated the possibility of whether any Frenet plane of a given space curve in a 3-dimensional Euclidean space $\mathbb{E}^{3}$ also is any Frenet plane of another space curve in the same space. We have obtained some characterizations of a given space curve by considering nine possible case.
\end{abstract}

\section{Introduction}

In the theory of curves in Euclidean space, one of the important and interesting problem is characterization of a regular curve. In the solution of the problem, the curvature functions $k_{1}$ (or $\varkappa$ ) and $k_{2}$ (or $\tau$ ) of a regular curve have an effective role. For example: if $k_{1}=0=k_{2}$, then the curve is a geodesic or if $k_{1}=$ constant $\neq 0$ and $k_{2}=0$, then the curve is a circle with radius $\left(1 / k_{1}\right)$, etc. Thus we can determine the shape and size of a regular curve by using its curvatures.

Another way in the solution of the problem is the relationship between the Frenet vectors of the curves (see [4]). For instance Bertrand curves:

In 1845, Saint Venant (see [4] and [6]) proposed the question whether upon the surface generated by the principal normal of a curve, a second curve can exist which has for its principal normal the principal normal of the given curve. This question was answered by Bertrand in 1850 in a paper which he showed that a necessary and sufficient condition for the existence of such a second curve is that a linear relationship with constant coefficients shall exist between the first and second curvatures of the given original curve. In other word, if we denote first and second curvatures of a given curve by $k_{1}$ and $k_{2}$ respectively, then for $\lambda, \mu \in \mathbb{R}$

Received December 13, 2013. Accepted January 24, 2014.

2010 Mathematics Subject Classification. 53A04.

Key words and phrases. Frenet planes, curvatures, circular helix, generalized helix, rectifying curve, Mannheim curve, Salkowski and anti-Salkowski curve.

${ }^{*}$ Corresponding author 
we have $\lambda k_{1}+\mu k_{2}=1$. Since the time of Bertrand's paper, pairs of curves of this kind have been called Conjugate Bertrand Curves, or more commonly Bertrand Curves.

Another interesting example is Mannheim curves: If there is exist a corresponding relationship between the space curves $\alpha$ and $\beta$ such that, at the corresponding points of the curves, the principal normal lines of $\alpha$ coincides with the binormal lines of $\beta$, then $\alpha$ is called a Mannheim curve, $\beta$ is called Mannheim partner curve of $\alpha$. Mannheim partner curves was studied by Liu and Wang (see [5]) in Euclidean 3-space and Minkowski 3-space.

Another way in the solution of the problem is position vector of a regular curve. In the Euclidean space $\mathbb{E}^{3}$, it is well-known that to each unit speed curve $\alpha: I \subset \mathbb{R} \rightarrow \mathbb{E}^{3}$ with at least four continuous derivatives, one can associate three mutually orthogonal unit vector fields $T$, $N$ and $B$ called respectively the tangent, the principal normal and the binormal vector fields. At each point $\alpha(s)$ of the curve $\alpha$, the planes spanned by $\{T, N\},\{T, B\}$ and $\{N, B\}$ are known respectively as the osculating plane, the rectifying plane and the normal plane. The curves $\alpha: I \subset \mathbb{R} \rightarrow \mathbb{E}^{3}$ for which the position vector $\alpha$ always lie in their rectifying plane, are for simplicity called rectifying curves. Similarly, the curves for which the position vector always lie in their normal plane, are for simplicity called normal curves. It is well known that the only normal curves in $\mathbb{E}^{3}$ are spherical curves. Finally, the curves for which the position vector $\alpha$ always lie in their osculating plane, are for simplicity called osculating curves. It is well known that the osculating curves in $\mathbb{E}^{3}$ are planar curves. In the Euclidean 3 -space, the rectifying curves are introduced by B. Y. Chen in ([1]). The Euclidean rectifying curves are studied in (see [1]). In particular, it is shown in (see [2]) that there exist a simple relationship between the rectifying curves and the centrodes, which play some important roles in mechanics, kinematics as well as in differential geometry in defining the curves of constant precession.

In this paper we ask the following question and investigate the possible answers of the question:

Is it possible that one of the Frenet planes of a given curve in $\mathbb{E}^{3}$ be a Frenet plane of a another space curve in the same space? Then we get many interesting results.

\section{Preliminaries}

Let $\alpha: I \subset \mathbb{R} \rightarrow \mathbb{E}^{3}$ be arbitrary curve in the Euclidean 3 -space $\mathbb{E}^{3}$. 
Recall that the curve $\alpha$ is said to be of unit speed (or parameterized by arclength function s) if $\left\langle\alpha^{\prime}(s), \alpha^{\prime}(s)\right\rangle=1$, where $\langle.,$.$\rangle is the standard$ scalar product of $\mathbb{E}^{3}$ given by

$$
\langle X, Y\rangle=x_{1} y_{1}+x_{2} y_{2}+x_{3} y_{3},
$$

for each $X=\left(x_{1}, x_{2}, x_{3}\right), Y=\left(y_{1}, y_{2}, y_{3}\right) \in \mathbb{E}^{3}$. In particular, the norm of a vector $X \in \mathbb{E}^{3}$ is given by $\|X\|=\sqrt{\langle X, X\rangle}$. Let $\{T, N, B\}$ be the moving Frenet frame along the unit speed curve $\alpha$, where $T, N$ and $B$ denote respectively the tangent, the principal normal and the binormal vector fields $([4])$.

\section{On The Curves And Frenet Planes In Euclidean 3-Space}

Let we consider the given two space curves $C$ and $\bar{C}$, defined on the same open interval $I \subset \mathbb{R}$. Let us attach moving triads $\{C, T, N, B\}$ and $\{\bar{C}, \bar{T}, \bar{N}, \bar{B}\}$ to $C$ and $\bar{C}$ at the corresponding points of $C$ and $\bar{C}$. We denote the arcs, curvatures and torsions of $C$ and $\bar{C}$ by $s, k_{1}, k_{2}$ and $\bar{s}, \overline{k_{1}}$, $\overline{k_{2}}$ respectively. At each point $C(s)$ of the curve $C$, the planes spanned by $\{T, N\},\{N, B\},\{T, B\}$ are known respectively as the osculating plane, the normal plane and the rectifying plane. We denote these planes by $O P, N P$ and $R P$, respectively. Now, we assume that $\bar{C}$ be a arbitrary unit speed space curve with curvatures $\overline{k_{1}}, \overline{k_{2}}$ and Frenet vectors $\bar{T}, \bar{N}$, $\bar{B}$. At each point $\bar{C}(\bar{s})$ of the curve $\bar{C}$, the planes spanned by $\{\bar{T}, \bar{N}\}$, $\{\bar{N}, \bar{B}\},\{\bar{T}, \bar{B}\}$ are known respectively as the osculating plane, the normal plane and the rectifying plane. We denote these planes by $\overline{O P}$, $\overline{N P}$ and $\overline{R P}$, respectively. Let $r=\frac{d \bar{s}}{d s}$. Then we have the following Frenet formulae:

$$
\begin{gathered}
T^{\prime}=k_{1} N, \quad N^{\prime}=-k_{1} T+k_{2} B, \quad B^{\prime}=-k_{2} N, \\
\bar{T}^{\prime}=r \overline{k_{1} N}, \quad \bar{N}^{\prime}=-r \overline{k_{1} T}+r \overline{k_{2} B}, \quad \bar{B}^{\prime}=-r \overline{k_{2} N},
\end{gathered}
$$

where $(')$ denotes $\frac{d}{d s}$.

In this section we ask the following question:

"Is it possible that one of the Frenet planes of a given curve be a Frenet plane of another space curve?" and we investigate the answer of the question. For this, we consider the following possible cases: 


\begin{tabular}{cccc} 
Case & Frenet plane of $C$ & $\frac{\text { Frenet plane of } \bar{C}}{\text { Condition }}$ \\
\cline { 2 - 4 } 2 & $s p\{T, N\}=O P$ & Cp $\{\bar{T}, \bar{N}\}=\overline{O P}$ & $O P=\overline{O P}$ \\
2 & $s p\{T, N\}=O P$ & $s p\{\bar{N}, \bar{B}\}=\overline{N P}$ & $O P=\overline{N P}$ \\
3 & $s p\{T, N\}=O P$ & $s p\{\bar{T}, \bar{B}\}=\overline{R P}$ & $O P=\overline{R P}$ \\
4 & $s p\{N, B\}=N P$ & $s p\{\bar{T}, \bar{N}\}=\overline{O P}$ & $N P=\overline{O P}$ \\
5 & $s p\{N, B\}=N P$ & $s p\{\bar{N}, \bar{B}\}=\overline{N P}$ & $N P=\overline{N P}$ \\
6 & $s p\{N, B\}=N P$ & $s p\{\bar{T}, \bar{B}\}=\overline{R P}$ & $N P=\overline{R P}$ \\
7 & $s p\{T, B\}=R P$ & $s p\{\bar{T}, \bar{N}\}=\overline{O P}$ & $R P=\overline{O P}$ \\
8 & $s p\{T, B\}=R P$ & $s p\{\bar{N}, \bar{B}\}=\overline{N P}$ & $R P=\overline{N P}$ \\
9 & $s p\{T, B\}=R P$ & $s p\{\bar{T}, \bar{B}\}=\overline{R P}$ & $R P=\overline{R P}$
\end{tabular}

Now we investigate these possible cases step by step:

Case 1. $O P=\overline{O P}$

In this case we investigate the answer of the following question:

"Is it possible that the osculating plane of a given space curve be the osculating plane of another space curve?".

We assume that, the osculating plane of the given curve $C$ be the osculating plane of a another space curve $\bar{C}$. Thus we have the following relation

$$
\bar{X}=X+a T+b N, \quad a \neq 0, b \neq 0
$$

where $\bar{X}$ and $X$ the position vectors of the curves $\bar{C}$ and $C$ respectively, and $a$ and $b$ are the non-zero functions of the parameter $s$. By taking the derivative of (3.3) with respect to $s$ and applying the Frenet formulas given in (3.1) and (3.2), we get

$$
\bar{T}=\left(1+a^{\prime}-b k_{1}\right) \frac{1}{r} T+\left(a k_{1}+b^{\prime}\right) \frac{1}{r} N+b k_{2} \frac{1}{r} B .
$$

Since $\bar{T} \in S p\{T, N\}$, we can write $\bar{T}=\lambda T+\mu N$, for some constant $\lambda$ and $\mu$. From (3.4) we have,

$$
\lambda T+\mu N=\left(1+a^{\prime}-b k_{1}\right) \frac{1}{r} T+\left(a k_{1}+b^{\prime}\right) \frac{1}{r} N+b k_{2} \frac{1}{r} B .
$$

Multiplying Eq.(3.5) by $B$, we obtain

$$
b k_{2} \frac{1}{r}=0 .
$$

Thus $b$ or $k_{2}$ must be zero which is controdiction with our assumption. Thus we give the following theorem: 
Theorem 1. There is no exist a pair of space curves $(C, \bar{C})$ in $\mathbb{E}^{3}$ for which osculating plane of $C$ is osculating plane of $\bar{C}$.

Case 2. $O P=\overline{N P}$

In this case we investigate the answer of the following question: "Can the osculating plane of a given space curve be the normal plane of another space curve?"

In this case we investigate the answer of the question. We assume that, the osculating plane of the given curve $C$ be the normal plane of a another space curve $\bar{C}$. Thus we have the following relation

$$
\bar{X}=X+a T+b N, \quad a \neq 0, b \neq 0,
$$

where $\bar{X}$ and $X$ the position vectors of the curves $\bar{C}$ and $C$ respectively, and $a$ and $b$ are the non-zero functions of the parameter $s$. By taking the derivative of (3.6) with respect to $s$ and applying the Frenet formulas given in (3.1) and (3.2), we get

$$
\bar{T}=\left(1+a^{\prime}-b k_{1}\right) \frac{1}{r} T+\left(a k_{1}+b^{\prime}\right) \frac{1}{r} N+b k_{2} \frac{1}{r} B .
$$

Since $B^{\perp}=S p\{T, N\}=S p\{\bar{N}, \bar{B}\}=\bar{T}^{\perp}, B$ is parallel to $\bar{T}$. First, multiplying Eq.(3.7) by $B$, we obtain

$$
b=\frac{r}{k_{2}} .
$$

Next, multiplying Eq.(3.7) by $N$, we have

$$
a=-\frac{1}{k_{1}}\left(\frac{1}{k_{2}} \frac{d^{2} \bar{s}}{d s^{2}}+\left(\frac{1}{k_{2}}\right)^{\prime} \frac{d \bar{s}}{d s}\right) .
$$

Thus we prove the following theorem.

Theorem 2. Let $C$ be a given unit speed curve in $\mathbb{E}^{3}$ with non-zero curvatures $k_{1}, k_{2}$ and Frenet vectors $T, N, B$. If the osculating plane of the curve $C$ is the normal plane of a another space curve $\bar{C}$, then $\bar{C}$ has the following form

$$
\bar{C}=C-\frac{1}{k_{1}}\left(\frac{1}{k_{2}} \frac{d^{2} \bar{s}}{d s^{2}}+\left(\frac{1}{k_{2}}\right)^{\prime} \frac{d \bar{s}}{d s}\right) T+\frac{1}{k_{2}} \frac{d \bar{s}}{d s} N .
$$

Conclusion 1. Without loss of generality, we assume that the curves $C$ and $\bar{C}$ have the same parameter $s$ and $k_{1}=k_{2}=$ constant $\neq 0$ then the curve $C$ is a spherical helix.

Case 3. $O P=\overline{R P}$ 
In this case we investigate the answer of the following question: "Can the osculating plane of a given space curve be the rectifying plane of a another space curve?"

In this case we investigate the answer of the question. We assume that, the osculating plane of the given curve $C$ be the rectifying plane of a another space curve $\bar{C}$. Thus we have the following relation

$$
\bar{X}=X+a T+b N, \quad a \neq 0, b \neq 0,
$$

where $\bar{X}$ and $X$ the position vectors of the curves $\bar{C}$ and $C$ respectively, and $a$ and $b$ are the non-zero functions of the parameter $s$. By taking the derivative of (3.9) with respect to $s$ and applying the Frenet formulas given in (3.1) and (3.2), we get

$$
\bar{T}=\left(1+a^{\prime}-b k_{1}\right) \frac{1}{r} T+\left(a k_{1}+b^{\prime}\right) \frac{1}{r} N+b k_{2} \frac{1}{r} B .
$$

Since $\bar{T} \in S p\{T, N\}$, we can write $\bar{T}=\lambda T+\mu N$, for some constant $\lambda$ and $\mu$. From (3.10) we have,

$$
\lambda T+\mu N=\left(1+a^{\prime}-b k_{1}\right) \frac{1}{r} T+\left(a k_{1}+b^{\prime}\right) \frac{1}{r} N+b k_{2} \frac{1}{r} B .
$$

Multiplying Eq. (3.11) by B, we obtain

$$
b k_{2} \frac{1}{r}=0 .
$$

Thus $b$ or $k_{2}$ must be zero which is controdiction with our assumption. Thus we give the following theorem:

Theorem 3. There is no exist a pair of space curves $(C, \bar{C})$ in $\mathbb{E}^{3}$ for which osculating plane of $C$ is rectifying plane of $\bar{C}$.

Case 4. $N P=\overline{O P}$

In this case we investigate the answer of the following question: "Can the normal plane of a given space curve be the osculating plane of a another space curve?".

In this case we investigate the answer of the question. Let assume that, the normal plane of the given curve $C$ be a the osculating plane of a another space curve $\bar{C}$. Thus we have the following relation

$$
\bar{X}=X+a N+b B, \quad a \neq 0, b \neq 0,
$$

where $\bar{X}$ and $X$ the position vectors of the curves $\bar{C}$ and $C$ respectively, and $a$ and $b$ are the non-zero functions of the parameter $s$. By taking the 
derivative of (3.15) with respect to $s$ and applying the Frenet formulas given in (3.1) and (3.2), we have

$$
\bar{T}=\left(1-a k_{1}\right) \frac{1}{r} T+\left(a^{\prime}-b k_{2}\right) \frac{1}{r} N+\left(a k_{2}+b^{\prime}\right) \frac{1}{r} B .
$$

Since $\bar{T} \in S p\{N, B\}$, we can write $\bar{T}=\lambda_{1} N+\mu_{1} B$, for some constant $\lambda_{1}$ and $\mu_{1}$. From (3.13) we get,

(3.14) $\lambda_{1} N+\mu_{1} B=\left(1-a k_{1}\right) \frac{1}{r} T+\left(a^{\prime}-b k_{2}\right) \frac{1}{r} N+\left(a k_{2}+b^{\prime}\right) \frac{1}{r} B$.

Multiplying Eq.(3.14), by $T$, we obtain

$$
a=\frac{1}{k_{1}} .
$$

By using (3.15) in (3.13) we get

$$
\bar{T}=\left(\left(\frac{1}{k_{1}}\right)^{\prime}-b k_{2}\right) \frac{1}{r} N+\left(\frac{k_{2}}{k_{1}}+b^{\prime}\right) \frac{1}{r} B .
$$

If we take derivative of (3.16) with respect to $s$ we obtain

$$
\begin{aligned}
\overline{k_{1}} \bar{N} r= & \left(-\left(\frac{1}{k_{1}}\right)^{\prime} k_{1}+b k_{1} k_{2}\right) \frac{1}{r} T \\
& +\left(\left(\frac{1}{k_{1}}\right)^{\prime \prime}-b^{\prime} k_{2}-b k_{2}^{\prime}-\frac{k_{2}^{2}}{k_{1}}-b^{\prime} k_{2}\right) \frac{1}{r} N \\
& +\left(2\left(\frac{1}{k_{1}}\right)^{\prime} k_{2}-b k_{2}^{2}+\frac{k_{2}^{\prime}}{k_{1}}+b^{\prime \prime}\right) \frac{1}{r} B \\
& +\left(\left(\left(\frac{1}{k_{1}}\right)^{\prime}-b k_{2}\right) N+\left(\frac{k_{2}}{k_{1}}+b^{\prime}\right) B\right)\left(\frac{1}{r}\right)^{\prime} .
\end{aligned}
$$

Since $\bar{N} \in S p\{N, B\}$, we can write in (3.17) $\bar{N}=\lambda_{2} N+\mu_{2} B$, where $\lambda_{2}$ and $\mu_{2}$ are constant. Multiplying Eq.(3.17) by $T$, we get

$$
b=-\frac{k_{1}^{\prime}}{k_{1}^{2} k_{2}} .
$$

If we put (3.15) and (3.18) in (3.12), we have,

$$
\bar{X}=X+\frac{1}{k_{1}} N-\frac{k_{1}^{\prime}}{k_{1}^{2} k_{2}} B .
$$

From (3.19), we can easily see that the curve $\bar{C}$ is a locus of the centers of the osculating spheres of the curve $C$. Thus we prove the following theorem: 
Theorem 4. Let $C$ and $\bar{C}$ be space curves with non-zero curvatures in $\mathbb{E}^{3}$. If the normal plane of the space curve $C$ is osculating plane of the curve $\bar{C}$ then $\bar{C}$ is the locus of the centers of the osculating spheres of the curve $C$.

Case 5. $N P=\overline{N P}$

In this case we investigate the answer of the following question: "Can the normal plane of a given space curve be the normal plane of a another space curve?".

In this case we investigate the answer of the question. We assume that, the normal plane of the given curve $C$ be the normal plane of a another space curve $\bar{C}$. Thus we have the following relation

$$
\bar{X}=X+a N+b B, \quad a \neq 0, b \neq 0,
$$

where $\bar{X}$ and $X$ the position vectors of the curves $\bar{C}$ and $C$ respectively, and $a$ and $b$ are the non-zero functions of the parameter $s$. By taking the derivative of (3.20) with respect to $s$ and applying the Frenet formulas given in (3.1) and (3.2), we get

$$
\bar{T}=\left(1-a k_{1}\right) \frac{1}{r} T+\left(a^{\prime}-b k_{2}\right) \frac{1}{r} N+\left(a k_{2}+b^{\prime}\right) \frac{1}{r} B .
$$

Since $T^{\perp}=S p\{N, B\}=S p\{\bar{N}, \bar{B}\}=\bar{T}^{\perp}$, we have $T$ is parallel to $T_{1}$ and

$$
<\bar{T}, T>=1 .
$$

multiplying Eq.(3.21)) by $T$, we have

$$
a=\frac{1}{k_{1}}(1-r) .
$$

Next multiplying Eq.(3.21) by $N$ we get

$$
b=\frac{1}{k_{2}}\left(\frac{1}{k_{1}}(1-r)\right)^{\prime} .
$$

Thus we give the following theorem:

Theorem 5. Let $C$ be a given unit speed curve in $\mathbb{E}^{3}$ with non-zero curvatures $k_{1}, k_{2}$ and Frenet vectors $T, N, B$. If the normal plane of the curve $C$ is the normal plane of a another space curve $\bar{C}$, then $\bar{C}$ has the following form

$$
\bar{C}=C+\frac{1}{k_{1}}\left(1-\frac{d \bar{s}}{d s}\right) N+\frac{1}{k_{2}}\left(\frac{1}{k_{1}}\left(1-\frac{d \bar{s}}{d s}\right)\right)^{\prime} B .
$$


Conclusion 2. Without loss of generality, we assume that the curves $C$ and $\bar{C}$ have the same parameter, then $C=\bar{C}$.

Case 6. $N P=\overline{R P}$

In this case we investigate the answer of the following question: "Can the normal plane of a given space curve be the rectifying plane of a another space curve?".

We assume that, the normal plane of the given curve $C$ be the rectifying plane of a another space curve $\bar{C}$. Thus we have the following relation

$$
\bar{X}=X+a N+b B, \quad a \neq 0, b \neq 0,
$$

where $\bar{X}$ and $X$ the position vectors of the curves $\bar{C}$ and $C$ respectively, and $a$ and $b$ are the non-zero functions of the parameter $s$. By taking the derivative of (3.24) with respect to $s$ and applying the Frenet formulas given in (3.1) and (3.2), we get

$$
\bar{T}=\left(1-a k_{1}\right) \frac{1}{r} T+\left(a^{\prime}-b k_{2}\right) \frac{1}{r} N+\left(a k_{2}+b^{\prime}\right) \frac{1}{r} B .
$$

Since $\bar{T} \in S p\{N, B\}$, we can write $\bar{T}=\lambda N+\mu B$, for some constant $\lambda$ and $\mu$. From (3.25) we have,

$$
\lambda N+\mu B=\left(1-a k_{1}\right) \frac{1}{r} T+\left(a^{\prime}-b k_{2}\right) \frac{1}{r} N+\left(a k_{2}+b^{\prime}\right) \frac{1}{r} B .
$$

Multiplying Eq. (3.26) by $T$, we obtain

$$
a=\frac{1}{k_{1}} .
$$

Differentiating equation (3.25) with respect to $s$ we have,

$$
\begin{aligned}
\overline{k_{1}} \bar{N} r= & -k_{1}\left(\left(\frac{1}{k_{1}}\right)^{\prime}-b k_{2}\right) \frac{1}{r} T+\left(\left(\frac{1}{k_{1}}\right)^{\prime \prime}-2 b^{\prime} k_{2}-b k_{2}^{\prime}-\frac{k_{2}^{2}}{k_{1}}\right) \frac{1}{r} N \\
& +\left(2\left(\frac{1}{k_{1}}\right)^{\prime} k_{2}-b k_{2}^{2}+\frac{k_{2}^{\prime}}{k_{1}}+b^{\prime \prime}\right) \frac{1}{r} B \\
& +\left(\left(\left(\frac{1}{k_{1}}\right)^{\prime}-b k_{2}\right) N+\left(\frac{k_{2}}{k_{1}}+b^{\prime}\right) B\right)\left(\frac{1}{r}\right)^{\prime} .
\end{aligned}
$$

Since $T^{\perp}=S p\{N, B\}=S p\{\bar{T}, \bar{B}\}=\bar{N}^{\perp}, T$ is parallel to $\bar{N}$ and multiplying Eq. (3.28) by $N$, we obtain

$$
b^{\prime}+\gamma b=\delta
$$

where $\gamma=\frac{k_{2}^{\prime}}{2 k_{2}}+\frac{r}{2}\left(\frac{1}{r}\right)^{\prime}$ and $\delta=\frac{1}{2 k_{2}}\left(\left(\frac{1}{k_{1}}\right)^{\prime \prime}-\frac{k_{2}^{2}}{k_{1}}\right)+\frac{r}{2 k_{2}}\left(\frac{1}{k_{1}}\right)^{\prime}\left(\frac{1}{r}\right)^{\prime}$. 
The solution of the equation(3.29)

$$
b(s)=e^{-\int \gamma d s}\left\{\int e^{\int \gamma d s} \delta d s+t\right\}, \text { where } t \in \mathbb{R} .
$$

Theorem 6. Let $C$ be a given unit speed curve in $\mathbb{E}^{3}$ with non-zero curvatures $k_{1}, k_{2}$ and Frenet vectors $T, N, B$. If the normal plane of the curve $C$ is the rectifying plane of a another space curve $\bar{C}$, then $\bar{C}$ has the following form

$$
\bar{C}=C+\frac{1}{k_{1}} N+\left(e^{-\int \gamma d s}\left\{\int e^{\int \gamma d s} \delta d s+t\right\}\right) B
$$

where $\gamma=\frac{k_{2}^{\prime}}{2 k_{2}}+\frac{r}{2}\left(\frac{1}{r}\right)^{\prime}$ and $\delta=\frac{1}{2 k_{2}}\left(\left(\frac{1}{k_{1}}\right)^{\prime \prime}-\frac{k_{2}^{2}}{k_{1}}\right)+\frac{r}{2 k_{2}}\left(\frac{1}{k_{1}}\right)^{\prime}\left(\frac{1}{r}\right)^{\prime}$ and $t \in \mathbb{R}$.

Conclusion 3. Without loss of generality, we assume that the curves $C$ and $\bar{C}$ have the same parameter $s(\bar{s}=s)$ and if $k_{1}=$ constant $\neq 0$ and $k_{2}=$ constant $\neq 0$, then we get

$$
b=-\frac{k_{2}}{2 k_{1}} s+t,
$$

for some $t \in \mathbb{R}$. In this case, it is clear that the curve $C$ is a circular helix.

Conclusion 4. Without loss of generality, we assume that the curves $C$ and $\bar{C}$ have the same parameter $s(\bar{s}=s)$ and if $k_{1}=$ constant $\neq 0$ and $k_{2} \neq 0, k_{2}$ nonconstant function then we get

$$
b=-\frac{1}{5} \frac{k_{2}^{2}}{k_{1}}+\frac{1}{\sqrt{k_{2}}} t
$$

for some $t \in \mathbb{R}$. In this case, the curve $C$ is a Salkowski curve in $\mathbb{E}^{3}$.

Conclusion 5. Without loss of generality, we assume that the curves $C$ and $\bar{C}$ have the same parameter $s(\bar{s}=s)$ and if $k_{2}=$ constant $\neq 0$ and $k_{1} \neq 0, k_{1}$ nonconstant function, then we get

$$
b=\frac{1}{2 k_{2}}\left(\frac{1}{k_{1}}\right)^{\prime}-\frac{k_{2}}{2} \int \frac{1}{k_{1}} d s+c,
$$

for some $c \in \mathbb{R}$. In this case, the curve $C$ is a anti- Salkowski curve in $\mathbb{E}^{3}$.

Case 7. $R P=\overline{O P}$ 
In this case we investigate the answer of the following question: "Can the rectifying plane of a given space curve be the osculating plane of a another space curve?".

Let assume that, the rectifying plane of the given curve $C$ be a the osculating plane of a another space curve $\bar{C}$. Thus we have the following relation

$$
\bar{X}=X+a T+b B, \quad a \neq 0, b \neq 0,
$$

where $\bar{X}$ and $X$ the position vectors of the curves $\bar{C}$ and $C$ respectively, and $a$ and $b$ are the non-zero functions of the parameter $s$. By taking the derivative of (3.30) with respect to $s$ and applying the Frenet formulas, we have

$$
\bar{T}=\left[\left(1+a^{\prime}\right) T+\left(a k_{1}-b k_{2}\right) N+b^{\prime} B\right] \frac{1}{r} .
$$

Since $\bar{T} \in S p\{T, B\}$, we can write $\bar{T}=\lambda T+\mu B$, for some constant $\lambda$ and $\mu$. From (3.31) we get,

$$
\lambda T+\mu B=\left[\left(1+a^{\prime}\right) T+\left(a k_{1}-b k_{2}\right) N+b^{\prime} B\right] \frac{1}{r} .
$$

Then multiplying Eq.(3.32) by $N$, we obtain

$$
\begin{gathered}
\frac{1}{r}\left(a k_{1}-b k_{2}\right)=0, \\
a k_{1}-b k_{2}=0 .
\end{gathered}
$$

Differentiating equation, (3.31) with respect to $s$ we have,

$$
\begin{aligned}
\overline{k_{1} N} r= & {\left[a^{\prime \prime} T+\left[k_{1}\left(1+a^{\prime}\right)-k_{2} b^{\prime}\right] N+b^{\prime \prime} B\right] \frac{1}{r} } \\
& +\left[\left(1+a^{\prime}\right) T+b^{\prime} B\right]\left(\frac{1}{r}\right)^{\prime} .
\end{aligned}
$$

Since $\bar{N} \in S p\{T, B\}$ we can write $\bar{N}=\lambda_{1} T+\mu_{1} B$, for some constant $\lambda_{1}$ and $\mu_{1}$. From (3.34) we get,

$$
\begin{aligned}
\overline{k_{1}}\left(\lambda_{1} T+\mu_{1} B\right) r= & {\left[a^{\prime \prime} T+\left[k_{1}\left(1+a^{\prime}\right)-k_{2} b^{\prime}\right] N+b^{\prime \prime} B\right] \frac{1}{r} } \\
& +\left[\left(1+a^{\prime}\right) T+b^{\prime} B\right]\left(\frac{1}{r}\right)^{\prime} .
\end{aligned}
$$

Multiplying Eq.(3.35) by $N$, we obtain

$$
\frac{1}{r}\left[k_{1}\left(1+a^{\prime}\right)-k_{2} b^{\prime}\right]=0 .
$$


Then we get

$$
k_{1}\left(1+a^{\prime}\right)-k_{2} b^{\prime}=0 .
$$

From equations (3.33) and (3.36), we get

$$
\frac{k_{1}}{k_{2}}=\frac{b}{a}=\frac{b^{\prime}}{1+a^{\prime}} .
$$

Thus we give the following results:

Conclusion 6. Let be $M$ and $\bar{M}$ be any points on the curves $C$ and $\bar{C}$ respectively. If we denote the position vectors $O M=X$ and $O \bar{M}=\bar{X}$, by using (3.30) and (3.31), we have

$$
\left\{\begin{array}{c}
M \bar{M}=a T+b B, \\
\bar{T}=\left(\left(1+a^{\prime}\right) T+b^{\prime} B\right) \frac{1}{r} .
\end{array}\right.
$$

From (3.37) and (3.38) we find that $M \bar{M}$ is parallel to $\bar{T}$. This means that the vector $M \bar{M}$ is tangent to the curve $\bar{C}$ at the point of the $\bar{M}$.

Conclusion 7. Since $\bar{M} \in S p\{T, B\}$, we can write

$$
M \bar{M}=\sin \theta T+\cos \theta B,
$$

where $\theta$ is denote the angle between the vector $M \bar{M}$ and $B$. So,

$$
\frac{a}{b}=\tan \theta \text {. }
$$

Also from (3.37) we get

$$
\tan \theta=\frac{k_{2}}{k_{1}} .
$$

Then the vector $M \bar{M}$ is parallel to Darboux vector of $C$ at the point $M$.

Definition 1. The vector $M \bar{M}$ is called rectifying line of the curve $C$ at the point $M \in C$.

Conclusion 8. The $\theta$ in Conclusion 7 is constant if and only if the curve $C$ is a general helix.

If we take the derivative of (3.33) and using (3.36) we obtain

$$
a k_{1}^{\prime}-b k_{2}^{\prime}=k_{1} \text {. }
$$

From (3.33) and (3.39) we get

$$
a=\frac{k_{1}}{k_{2}} \frac{1}{\left(\frac{k_{1}}{k_{2}}\right)^{\prime}},
$$


and

$$
b=-\frac{1}{\left(\frac{k_{2}}{k_{1}}\right)^{\prime}} .
$$

Also from (3.40) and (3.41) we can easily obtain that

$$
\|M \bar{M}\|^{2}=\frac{k_{1}^{2}\left(k_{1}^{2}+k_{2}^{2}\right)}{k_{2} k_{1}^{\prime}-k_{1} k_{2}^{\prime}} .
$$

Conclusion 9. If $a=\frac{k_{1}}{k_{2}} \frac{1}{\left(\frac{k_{1}}{k_{2}}\right)}=$ constant then we get

$$
\frac{k_{1}}{k_{2}}=c_{2} e^{c_{1} s} .
$$

Conclusion 10. If $b=-\frac{1}{\left(\frac{k_{2}}{k_{1}}\right)^{\prime}}=$ constant then we get

$$
\frac{k_{2}}{k_{1}}=c s+d, c \in \mathbb{R}, d \in \mathbb{R},
$$

which means that the curve $C$ is a rectifiying curve.

We note that such curves also called canonical geodesics by Izumiya and Takeuchi in [3].

Conclusion 11. If $\|M \bar{M}\|^{2}=\frac{k_{1}^{2}\left(k_{1}^{2}+k_{2}^{2}\right)}{k_{2} k_{1}^{\prime}-k_{1} k_{2}^{\prime}}=$ constant then we get

$$
\frac{k_{2}}{k_{1}}=\sinh \left(c_{1} s+c_{2}\right) \text {, }
$$

which means that the curve $C$ is a Mannheim curve (see [5]).

Case 8. $R P=\overline{N P}$

In this case we investigate the answer of the following question: "Can the rectifying plane of a given space curve be the normal plane of another space curve?".

We assume that, the rectifying plane of the given curve $C$ be the normal plane of a another space curve $\bar{C}$. Thus we have the following relation

$$
\bar{X}=X+a T+b B, \quad a \neq 0, b \neq 0,
$$

where $\bar{X}$ and $X$ the position vectors of the curves $\bar{C}$ and $C$ respectively, and $a$ and $b$ are the non-zero functions of the parameter $s$. By taking the 
derivative of (3.43) with respect to $s$ and applying the Frenet formulas, we get

$$
\bar{T}=\left[\left(1+a^{\prime}\right) T+\left(a k_{1}-b k_{2}\right) N+b^{\prime} B\right] \frac{1}{r} .
$$

Since $N^{\perp}=S p\{T, B\}=S p\{\bar{N}, \bar{B}\}=\bar{T}^{\perp}, N$ is parallel to $\bar{T}$. Multiplying Eq.(3.44) by $N$ we obtain

$$
a k_{1}-b k_{2}=r
$$

First multiplying Eq.(3.44) by $T$, next multiplying Eq.(3.44) by $B$, we obtain

$$
\begin{gathered}
a=-s+c_{1}, \\
b=c_{2},
\end{gathered}
$$

for some constant $c_{1}$ and $c_{2}$ respectively. Thus we have

$$
\left(-s+c_{1}\right) k_{1}-c_{2} k_{2}=r \text {. }
$$

Theorem 7. Let $C$ be a given unit speed curve in $\mathbb{E}^{3}$ with non-zero curvatures $k_{1}, k_{2}$ and Frenet vectors $T, N, B$. If the rectifying plane of the curve $C$ is the normal plane of a another space curve $\bar{C}$, then $\bar{C}$ has the following form

$$
\bar{C}=C+\left(-s+c_{1}\right) T+c_{2} B
$$

where $c_{1}, c_{2} \in \mathbb{R}$

Case 9. $R P=\overline{R P}$

In this case we investigate the answer of the following question: "Can the rectifying plane of a given space curve be the rectifying plane of another space curve?".

We assume that, the rectifying plane of the given curve $C$ be the rectifying plane of a another space curve $\bar{C}$. Thus we have the following relation

$$
\bar{X}=X+a T+b B, \quad a \neq 0, b \neq 0,
$$

where $\bar{X}$ and $X$ the position vectors of the curves $\bar{C}$ and $C$ respectively, and $a$ and $b$ are the non-zero functions of the parameter $s$. By taking the derivative of (3.46) with respect to $s$ and applying the Frenet formulas, we get

$$
\bar{T}=\left[\left(1+a^{\prime}\right) T+\left(a k_{1}-b k_{2}\right) N+b^{\prime} B\right] \frac{1}{r} .
$$


Since $\bar{T} \in S p\{T, B\}$, we can write $\bar{T}=\lambda T+\mu B$, for some constant $\lambda$ and $\mu$. From (3.47) we get,

$$
\lambda T+\mu B=\left[\left(1+a^{\prime}\right) T+\left(a k_{1}-b k_{2}\right) N+b^{\prime} B\right] \frac{1}{r} .
$$

Multiplying Eq.(3.48) by $N$, we obtain

$$
a k_{1}-b k_{2}=0 .
$$

Differentiating equation (3.47) with respect to $s$ we have,

$$
\begin{aligned}
\overline{k_{1} N r} r= & {\left[a^{\prime \prime} T+\left[k_{1}\left(1+a^{\prime}\right)-k_{2} b^{\prime}\right] N+b^{\prime \prime} B\right] \frac{1}{r} } \\
& +\left[\left(1+a^{\prime}\right) T+b^{\prime} B\right]\left(\frac{1}{r}\right)^{\prime} .
\end{aligned}
$$

Since $N^{\perp}=S p\{T, B\}=S p\{\bar{T}, \bar{B}\}=\bar{N}^{\perp}, N$ is parallel to $\bar{N}$. First multiplying Eq.(3.50) by $T$, next multiplying Eq.(3.50) by $B$, we obtain

$$
\begin{gathered}
a^{\prime \prime} \frac{1}{r}+\left(1+a^{\prime}\right)\left(\frac{1}{r}\right)^{\prime}=0, \\
b^{\prime \prime} \frac{1}{r}+b^{\prime}\left(\frac{1}{r}\right)^{\prime}=0,
\end{gathered}
$$

respectively. Thus we have

$$
\begin{gathered}
a=-s+c_{1} \int r d s+c_{2}, \\
b=d_{1} \int r d s+d_{2},
\end{gathered}
$$

for some constant $c_{1}, c_{2}, d_{1}$, and $d_{2}$.

Theorem 8. Without loss of generality, we assume that the curves $C$ and $\bar{C}$ have the same parameter $s(\bar{s}=s)$. Let $C$ be a given unit speed curve in $\mathbb{E}^{3}$ with non-zero curvatures $k_{1}, k_{2}$ and Frenet vectors $T$, $N, B$. If the rectifying plane of the curve $C$ is the rectifying plane of a another space curve $\bar{C}$, then $\bar{C}$ has the following form

$$
\bar{C}=C+\left(c_{1} s+c_{2}\right) T+\left(d_{1} s+d_{2}\right) B .
$$

where $c_{1}, c_{2}, d_{1}, d_{2} \in \mathbb{R}$.

From (3.49) we get

$$
\frac{k_{2}}{k_{1}}=\frac{c_{1} s+c_{2}}{d_{1} s+d_{2}} .
$$

Thus we give the following results: 
Conclusion 12. If $c_{1} d_{2}-c_{2} d_{1}=0$, then $\frac{k_{1}}{k_{2}}=$ constant. In this case the curve of $C$ is a circular helix or a generalized helix in $\mathbb{E}^{3}$.

Example 1. Let we consider the circular helix $C(s)=(\cos s, \sin s, s)$ in $\mathbb{E}^{3}$. It is easily obtained that the Frenet vectors and curvatures of the curve as follows:

$$
\begin{aligned}
& T(s)=\quad\left(\frac{-\sin s}{\sqrt{2}}, \frac{\cos s}{\sqrt{2}}, \frac{1}{\sqrt{2}}\right) \\
& N(s)=(-\cos s,-\sin s, 0) \\
& B(s)=\quad\left(\frac{\sin s}{\sqrt{2}}, \frac{-\cos s}{\sqrt{2}}, \frac{1}{\sqrt{2}}\right) \\
& k_{1}=\frac{1}{\sqrt{2}} \quad k_{2}=\frac{1}{\sqrt{2}} .
\end{aligned}
$$

Now we assume that there exist a space curve $\bar{C}(s)$ which is have the same rectifying plane with $C$. Then by using the equations (3.5) and (3.10), and taking $c_{1}=d_{1}=0, c_{2}=d_{2}=\sqrt{2}$, we obtained that $\bar{C}(s)=$ $(\cos s, \sin s, s+2)$. It is easily shown that $C(s)$ and $\bar{C}(s)$ are congruent curves according to the Fundamental theorem of curves in $\mathbb{E}^{3}$.

Conclusion 13. If we take $d_{2}=0$ in (3.51) then we get $\frac{k_{1}}{k_{2}}=\frac{c_{1}}{d_{1}}+$ $\frac{c_{2}}{d_{1}} \frac{1}{s}$. In this case the curve of $C$ is a rectifying curve up to parametrizations of the curve $C$.

\section{Acknowledgement}

We wish to thank the referee for the careful reading of the manuscript and constructive comments that have substantially improved the presentation of the paper.

\section{References}

[1] Chen, B. Y., When does the position vector of a space curve always lie in its rectifying plane?, Amer. Math. Monthly 110 (2003), 147-152.

[2] Chen, B. Y. and Dillen, F., Rectifying curves as centrodes and extremal curves, Bull. Inst. Math. Academia Sinica 33(2) (2005), 77-90.

[3] Izumiya, S. and Takeuchi, N., New special curves and developable surfaces, Turkish J. Math. 28(2) (2004), 153-163.

[4] Kuhnel, W., Differential geometry: curves-surfaces-manifolds, Braunschweig, Wiesbaden, 1999.

[5] Liu, H. and Wang, F., Mannheim partner curves in 3-space, Journal of Geometry, 88 (2008), 120-126.

[6] Millman, R. S. and Parker, G. D., Elements of differential geometry, Prentice-Hall, New Jersey, 1977. 
Siddika Özkaldi Karakuş

Department of Mathematics, Faculty of Sciences and Arts, Bilecik Şeyh Edebali University, Bilecik-TURKEY.

E-mail : sozkaldi98@yahoo.com

Kazim İlarslan

Department of Mathematics, Faculty of Science and Arts,

University of Kırıkkale, Kırıkkale-TURKEY.

E-mail : kilarslan@yahoo.com

Yusuf Yayli

Department of Mathematics, Faculty of Science, University of Ankara, Tandoğan, Ankara-TURKEY.

E-mail : yayli@science.ankara.edu.tr 